\title{
Pesquisa
}

\section{ANÁLISE FATORIAL DA ESCALA DE AVALIAÇÃO DA FADIGA EM UMA AMOSTRA DE UNIVERSITÁRIOS DE INSTITUIÇÃO PÚBLICA}

\author{
Gislene Farias de Oliveira (1) \\ Valdiney Veloso Gouveia (2) \\ Ginna Pereira Peixoto (3) \\ Maria Alinele Lucena Soares (4)
}

\begin{abstract}
Resumo
Este estudo tem como objetivo comprovar a estrutura fatorial da Escala de Avaliação da Fadiga numa amostra de 200 estudantes universitários de instituições públicas de ensino, em João Pessoa/PB. Estes responderam a Escala de Avaliação da Fadiga, composta por 10 itens avaliados em escala de cinco pontos, variando de $\mathbf{1}=$ Nunca a $\mathbf{5}=$ Sempre. O pacote estatístico SPSSWIN 11.5 (Statistical Package for the Social Sciences) foi utilizado para tabulação e análise dos dados. A partir do $K M O=0,82$ e do Teste de Esfericidade de Bartlett=603,84; $p<0,000$, verificou-se a pertinência da realização da análise fatorial. Em seguida, fixando-se a extração de um fator, procedeu-se a análise fatorial dos componentes principais. Os resultados desta análise corroboram a presença de um fator, explicando conjuntamente $39,2 \%$ da variância total. Foram excluídos dois itens dos 10 originais por não terem apresentado carga fatorial satisfatória $( \pm 0,30)$. Na escala final o fator (Fadiga) ficou composto por 8 itens, com cargas fatoriais variando entre 0,46 a $0,78(\alpha=0,81)$. Conclui-se, portanto, que esta medida de Fadiga apresenta parâmetros psicométricos satisfatórios representando adequadamente o construto em questão.
\end{abstract}

Palavras-chave: Escala de Fadiga, Fadiga Crônica, Avaliação da Fadiga

\section{Introdução}

O presente trabalho constitui-se de um estudo complementar que faz parte de uma pesquisa mais ampla, na qual visamos identificar o Bem-estar dos profissionais de saúde. A Avaliação da fadiga será utilizada como um dos instrumentos para a avaliação do Bem-estar. Nosso interesse neste estudo centrou-se na validação da Escala de Avaliação da Fadiga, de maneira a identificar sua distribuição fatorial no contexto de estudantes universitários de áreas de saúde, como pré-condição para utilização do instrumento em profissionais de áreas de saúde.

O aumento mundial na utilização de sistemas de trabalho em turnos, principalmente nas áreas de saúde, explica o crescimento no número de estudos que enfocam os reflexos na saúde dos trabalhadores expostos (Smith et al., 1998)

A síndrome da fadiga crônica é uma doença caracterizada pela presença de fadiga inexplicável, de duração maior que seis meses, que envolve sintomas como cefaléia, dores pelo corpo, 
dor nas articulações, problemas com a memória e distúrbio do sono, causando incapacidade física ao paciente. A fadiga crônica é uma condição muito comum na população geral. (Levy et al., 1998)

Estudos mostram que a queixa de fadiga como sintoma está presente em 21 a $38 \%$ das pessoas ocidentais. Já a prevalência da síndrome da fadiga crônica, baseada nos critérios diagnósticos do CDC - Center for Disease Control, Atlanta - EUA, está em torno de 0,5\% da população em geral. Tais critérios foram estabelecidos em 1988 e revisados em 1994. Não é incomum a doença surgir em períodos em que estão presentes desordens afetivas, como ansiedade e depressão. Mais de 30\% dos pacientes fadigados apresentam problemas apenas na esfera psicológica. Por outro lado, a depressão pode acompanhar a síndrome da fadiga crônica desde o seu inicio ou se manifestar em decorrência desta. (Varela, 2009).

A presença de tantos sintomas da Síndrome da Fadiga Crônica foi explicada fisiologicamente como uma lesão situada na região límbica do cérebro. O mesmo trabalho sugere também que várias seriam as etiologias da Síndrome da Fadiga Crônica, desde que a lesão esteja presente na região límbica do cérebro, seja ela desmielizante, infecciosa, neoplásica, paraneoplásica ou anóxica (Goldstein, 1993).

Fadiga é uma experiência universal que geralmente desaparece após uma boa noite de repouso, em muitos casos, esta sensação de desconforto pode persistir tornando-se crônica (Piper, 1986).

No caso de indivíduos com doenças crônicas, a fadiga se apresenta de maneira diferente da fadiga diária, este tipo se apresenta como um sintoma complexo provocado por condições físicas e psicológicas preexistentes. De maneira geral, inclusive nas doenças crônicas, a fadiga é um sintoma comumente angustiante. (Rhodes et al.,1988).

A partir da década de 20 as tentativas de conceituar fadiga reportam dificuldades de aferição e interpretação por se tratar de um fenômeno bastante complexo (Marziale,1990).

Potempa (1993) relata que fadiga é uma queixa comum relacionada com eventos específicos como pouco sono ou falta de exercícios, nesses casos a fadiga é facilmente remediável. Entretanto, sua persistência interfere no cotidiano do indivíduo, tornando-o debilitado. Essa condição é freqüentemente referida como fadiga crônica.

Ryan (1994) define a fadiga como um estado geral do indivíduo, resultante da atividade contínua de trabalho.

De acordo com Cameron (1973), fadiga é uma resposta generalizada ao estresse com efeitos agudos ou crônicos. O autor evidencia a importância da ansiedade e dos distúrbios do sono como diretamente relacionados ao aparecimento da fadiga.

Segundo Lino (1978), a fadiga é uma alteração reversível da coordenação das funções biológicas, físicas e psíquicas. Uma vez retiradas as causas que a provocam, o organismo se põe em situação normal. Em caso de persistência, a fadiga, pode tornar-se patológica. 
Os sinônimos de fadiga segundo Aurélio Buarque de Holanda Ferreira: cansaço, canseira, fadigamento e diminuição gradual da resistência de um material por efeito de solicitações repetidas (Ferreira, 1999).

A fadiga, pode ainda ser definida como uma sensação subjetiva de exaustão influenciada pelo ritmo circadiano, podendo variar quanto a sua duração, freqüência e grau.(Piper, 1986)

Assim como existem vários conceitos em torno da fadiga, existem também vários sistemas de classificação, mas muitos deles são de pouca validade para a psicologia. Porém, o conceito de fadiga crônica e aguda, que caracteriza o sintoma de acordo com sua duração, é de alguma valia. Segundo Piper (1986), na forma aguda, a fadiga é um mecanismo protetor, mas quando se torna excessiva ou constante (crônica) deixa de ser um mecanismo protetor e pode levar a uma fuga de atividade.

Os indicadores da fadiga, citados na literatura, podem ser objetivos como os fisiológicos, bioquímicos e comportamentais, e subjetivos que são indicadores percebidos como sentimentos e sensações específicas de cansaço (Skalla e Lacasse, 1992; Yoshitake, 1978; Yoshitake, 1992).

Diante do exposto, este trabalho propôs-se a validar a Escala de Avaliação da Fadiga em uma amostra de estudantes universitários de três áreas da saúde. Analisaremos os dados a partir da classificação de fadiga aguda ou crônica e seus indicadores subjetivos. Utilizaremos o instrumento proposto por (Michielsen et al., 2004).

\section{Histórico}

O instrumento utilizado para avaliar Fadiga, elaborado por Michielsen, H.J., De Vires, J. Heck, G.L.V.,Vijver, F. J. R. V., Sijtsma, K. em 2004. Inicialmente, a escala foi administrados em uma amostra de 876 sujeitos de várias ocupações profissionais. 452 homens (com idade média de 41 anos) e 412 mulheres (com idade média de 39 anos) Um fator emergiu e, por meio de uma avaliação criteriosa, foram eliminados os itens que não possuíam peso fatorial superior a 0,40. Após aplicação em uma nova amostra de 1893 sujeitos, sendo 1.128 homens (com idade média de 46 aos) e 765 mulheres (com idade média de 42 aos) de perfil igual ao anterior, a consistência interna dos itens se mostrou satisfatória, pois apresentou um alfa de Cronbach de 0,87 explicando em $48 \%$ a variância (Michielsen et al., 2004).

A Escala de Avaliação da Fadiga avalia como a pessoa vivencia os sintomas da fadiga no seu dia-a-dia. A intensidade e a frequiência das respostas é medida com uma escala de pontuação que varia de 1 a 5 .

É um instrumento utilizado exclusivamente para a avaliação da síndrome, não levando em consideração os elementos antecedentes e as consequiências de seu processo. Ela avalia índices de Fadiga de acordo com apuração de escores, sendo que altos escores significam alto nível de Fadiga e, baixos escores significam baixo nível de Fadiga. 


\section{Método}

A amostra se constituiu de 200 estudantes universitários da Universidade Federal da Paraíba, sendo 37,8\% de Psicologia, 34,3\% de Medicina e 27,9\% de Enfermagem. A maioria do sexo feminino $(64,2 \%)$, casados $(90 \%)$, que se identificaram como católicos $(59,7 \%)$ e protestantes $(16,4 \%)$, pertencentes à classe média baixa $(28,4 \%)$ e classe média alta $(27,4 \%)$, com idades variando entre 18 e $39 \operatorname{anos}(M=22$ anos; $D P=3,43)$.

A Escala de Avaliação da Fadiga, é auto-aplicável e totaliza 10 itens. As respostas são avaliadas em escala de cinco pontos, variando de $\mathbf{1}$ para Nunca e $\mathbf{2}$ para Raramente, $\mathbf{3}$ para Algumas vezes, $\mathbf{4}$ para Frequentemente e $\mathbf{5}$ para Sempre.

Foi verificado que os sujeitos não apresentaram dificuldade em responder os itens do instrumento.

Com relação aos procedimentos para a coleta de dados, primeiramente foi realizado um contato com os sujeitos na instituição de ensino, quando foi apresentado o objetivo do estudo a fim de obter a autorização para a aplicação do instrumento. Este foi entregue aos alunos em suas classes, antes do início das aulas, tendo sido a coleta realizada, utilizando-se um envelope único na mesa do professor.

A pesquisa teve aprovação do Comitê de Ética da Universidade Federal da Paraíba, tendo sido realizados os procedimentos conforme resolução 196 do Conselho Nacional de Saúde (CNS), no que diz respeito à pesquisa com seres humanos (Hutz e Spink, 1996). O banco de dados foi digitado e, posteriormente, analisado através do pacote estatístico SPSSWIN (Statistical Package for the Social Sciences) versão 11.5. A validação da Escala de Avaliação da Fadiga foi feita através de medidas de adequação da amostra à análise fatorial, validade de construto pelo método de componentes principais e confiabilidade pelo método do alfa de Cronbach.

\section{Resultados}

Antes da realização da análise fatorial foram contemplados alguns critérios, necessários à sua realização. A adequação da amostra foi mensurada pelos seguintes critérios: determinante da matriz de correlação, medida de adequação da amostra de Kaiser-Meyer-Olkin (KMO), teste de esfericidade de Bartlett e medidas individuais de adequação da amostra (Tabela 1).

A intercorrelação foi confirmada pelo teste de esfericidade de Bartlett $\left(X^{2}=603,845 ; \mathrm{p}<0,000\right)$. Tais valores rejeitam a hipótese nula de que não haja intercorrelação entre os valores. Ao contrário, 
existem intercorrelações estatisticamente significativas, isso indica que a matriz de dados é adequada para proceder à análise fatorial.

Tabela 1 - KMO and Bartlett's Test

\begin{tabular}{|cc|c|}
\hline \multicolumn{2}{|c|}{ Kaiser-Meyer-Olkin Measure of Sampling } &, 829 \\
Adequacy. & \\
Bartlett's Test of $\quad$ Approx. Chi-Square & $\mathbf{6 0 3 , 8 4 5}$ \\
Sphericity & DF & 45 \\
& Sig. & $\mathbf{, 0 0 0}$ \\
\hline
\end{tabular}

Para realização da análise fatorial incluímos os 10 itens da Escala de Avaliação da Fadiga, que se encontram descritos em sua distribuição fatorial na matriz estrutural (Tabela 2). Assumindo-se, que os fatores são independentes entre si, foi utilizado o método de extração de fatores usando componentes principais, com rotação ortogonal Varimax. Os resultados desta análise corroboram a presença de um fator, explicando conjuntamente $39,2 \%$ da variância total.

Tabela 2 - Total Variance Explained

\begin{tabular}{|l|l|l|l|l|l|c|}
\hline \multirow{2}{*}{ Component } & \multicolumn{3}{|l|}{ Initial Eigenvalues } & \multicolumn{3}{l|}{ Extraction Sums of Squared Loadings } \\
\cline { 2 - 6 } & Total & $\begin{array}{c}\text { \% of } \\
\text { Variance }\end{array}$ & $\begin{array}{c}\text { Cumulative } \\
\%\end{array}$ & Total & $\begin{array}{c}\text { \% of } \\
\text { Variance }\end{array}$ & $\begin{array}{c}\text { Cumulative } \\
\%\end{array}$ \\
\hline 1 & 3,924 & 39,241 & 39,241 & 3,924 & $\mathbf{3 9 , 2 4 1}$ & 39,241 \\
2 & 1,485 & 14,847 & 54,088 & & & \\
3 & 1,037 & 10,369 & 64,456 & & & \\
4 &, 757 & 7,569 & 72,025 & & & \\
5 &, 600 & 6,003 & 78,028 & & & \\
6 &, 555 & 5,547 & 83,575 & & & \\
7 &, 509 & 5,093 & 88,669 & & & \\
9 &, 491 & 4,913 & 93,582 & & & \\
10 &, 355 & 3,548 & 97,130 & & & \\
\hline
\end{tabular}

O fator encontrado através da análise fatorial da Escala de Avaliação da Fadiga em nossa amostra confirmam, semanticamente, os itens da Fadiga propostos por Michielsen et al. (2004). Foram excluídos dois itens dos dez originais por não terem apresentado carga fatorial satisfatória 
$( \pm 0,30)$. Na escala final o fator (Fadiga) ficou composto por 8 itens, com cargas fatoriais variando entre 0,46 a 0,78 (Tabela 3 ).

Tabela 3 - Component Matrix(a)

\begin{tabular}{|c|c|}
\hline & $\begin{array}{c}\text { Compone } \\
\mathrm{nt}\end{array}$ \\
\cline { 2 - 2 } & 1 \\
\hline f05 &, 782 \\
$\mathrm{f} 02$ &, 776 \\
f09 &, 763 \\
Sinto-me & \\
incomodado devido à &, 721 \\
fadiga &, 662 \\
f08 &, 579 \\
f07 &, 572 \\
f04 & \\
Posso me concentrar &, 480 \\
bem quando estou \\
fazendo algo \\
f06 \\
f03
\end{tabular}

Extraction Method: Principal Component Analysis.

a 1 components extracted.

Na Tabela 4 e Tabela 5 se apresentam respectivamente a média, o desvio-padrão e o valor do Alfa de Crombach $(\alpha=0,81)$. para o fator, além das correlações obtidas. A média foi de 33,62 ( $d p=$ 104,16).

Tabela 4 - Scale Statistics

\begin{tabular}{|l|l|l|l|}
\hline Mean & Variance & $\begin{array}{l}\text { Std. } \\
\text { Deviation }\end{array}$ & $\begin{array}{l}\text { of } \\
\text { Items }\end{array}$ \\
\hline 33,62 & 104,165 & 10,206 & 8 \\
\hline
\end{tabular}




\section{Tabela 5 - Reliability Statistics}

\begin{tabular}{|l|l|}
\hline $\begin{array}{c}\text { Cronbach's } \\
\text { Alpha }\end{array}$ & $\begin{array}{c}\text { N of } \\
\text { Items }\end{array}$ \\
\hline, 818 & 8 \\
\hline
\end{tabular}

Estes resultados indicam a fidedignidade da Escala de Avaliação da Fadiga em nossa amostra, demonstrando a capacidade desta na medição da Fadiga em universitários.

A partir dos resultados obtidos conclui-se que esta medida de Fadiga apresenta parâmetros psicométricos satisfatórios representando adequadamente o construto em questão.

\section{Discussão}

Resultados obtidos nesta amostra confirmam o modelo proposto para a Escala de Avaliação da Fadiga no que diz respeito à constituição de uma dimensão, como propõe a Escala original (Michielsen et al., 2004).

O fator explica, neste estudo, 39,2\% da variância, resultado bastante semelhante ao encontrado no estudo original de Michielsen et al. (2004), o qual identificou 48,0\% da variância explicada.

O teste de fidedignidade avaliado pelo Alfa de Cronbach, neste estudo, constatou que a Escala de Avaliação da Fadiga apresenta uma boa consistência interna (alfa >0,81).

\section{Conclusão}

Como o instrumento avalia uma síndrome, é preciso considerar uma série de aspectos que ainda podem ser inseridos como itens na Escala de Avaliação da Fadiga. Entretanto, no atual estado da arte dos estudos sobre Fadiga, a Escala de Avaliação da Fadiga apresenta-se como uma escala válida e fidedigna nas diferentes realidades onde a síndrome tem sido estudada.

Considerando a amostra de universitários utilizada no presente estudo, podemos afirmar que a versão aqui proposta apresenta os requisitos necessários em termos de consistência interna e validade fatorial para ser também utilizada na avaliação da síndrome de Fadiga em profissionais de saúde. 


\section{Referências}

Cameron, C. (1973). Theory of fatigue. Ergonomics, v. 16, n. 5, p. 633-648. Ferreira, A.B.de H. (1999). Novo Aurélio Século XXI. O dicionário da língua portuguesa. 3. ed. Rio de Janeiro: Nova Fronteira. 2132 p.

Goldstein, G. (1993). Chronic Fatigue Syndrome. The Limbic Hypothesis. The Haworth Press Inc., N York.

Hutz, C. S. \& Spink, M. J. (1996). Orientações éticas para psicólogos envolvidos em pesquisas com seres humanos. Disponível em <http://www.psicologia.urgrs.etica2.htm >. Acesso em 09/03/2005.

Levy, J. A. Fadiga versus Síndrome da Fadiga Crônica. Disponível em $<$ http://www.emedix.com.br/ >. Acesso em 13/10/2005.

Levy JA; Arouca EG; Cerqueira LA. (1998). Síndrome da Fadiga Crônica. An Paul Med Cir (1998). p. 31-34.

Levy, JA; Vieira, JC. ( ) - Fadiga Crônica. In Fraguas Jr. R, Figueiró J. Depressões em Medicina Interna, Atheneu, S. Paulo, p. 419-426.

Lino, B.H.(1978). A fadiga. In: CONGRESSO NACIONAL DE PREVENÇÃO DE ACIDENTES DE TRABALHO, 17. Anais. São Paulo: FUNDACENTRO. p. 101-114.

Marziale, M.H.P. (1990). Estudo da fadiga mental de enfermeiros atuantes em instituição hospitalar com esquema de trabalho em turnos alternantes. Ribeirão Preto, 1990. p.122. Dissertação (Mestrado) da Faculdade de Filosofia Ciências e Letras de Ribeirão Preto, Universidade de São Paulo.

Mayo, E. (1959). Problemas humanos de una civilización industrial. Coleccion el hombre, la sociedad y la história. Buenos Aires.

Michielsen, H.J., De Vires, J. Heck, G.L.V.,Vijver, F. J. R. V., Sijtsma, K. (2004). Examination of the dimensionality of fatigue: the construction of the fatigue assessment scale (FAS). European Journal Psychological Assessment. Vol 20. Issue 1. pp. 39-48.

Piper, B.F. (1986). Fatigue. In: Carrieri, V.K.; Lindsey, A.M.; West, C.W. (eds.) Pathophysiological phenomena in nursing: human responses to illness. Philadelphia: W.B. Saunders. p. 219-234.

Potempa, K.M. (1993). Chronic fatigue. Annu.Rev.Nurs.Res., v. 11, p. 57-76.

Rhodes, V.; Watson, P.; Hanson, B. (1988). Patients' descriptions of the influence of tiredness and weakness on self-care abilities. Cancer Nurs., v. 11, p. 186-194. Ryan, A.H.(1994). Varieties of fatigue. Am.J.Psychol., v. 57, p. 565-569.

Smith L, Hammond T, Macdonald I, Folkard S. 12 h shifts are popular but are they a solution? Int J Ind Erg 1998;21:323-31.

Skalla, K.A.; Lacasse, C. (1992). Fatigue and the patient with cancer: what is it and what can I do about it ?. Oncol.Nurs.Forum, v. 19, n. 10, p. 1540-1541. 
Varella, D. (2009). Síndrome da fadiga crônica. Disponível em: http://www.drauziovarella.com.br/artigos/fadiga.asp. Acesso em 30 out 2009.

Volle, M.; Brisson, G.R.; Dion, M.; Tanaka, M. (1978). Travail fatigue et fréquence de fusion critique usuelle. Ergonomics, v. 21, n. 7, p. 551-558.

Yoshitake, H. (1969). Rating the feelings of the fatigue. J.Science Labour, v. 45, n. 7, p. 422-432.

Yoshitake, H. (1978). There are characteristic patterns of subjective fatigue symptoms. Ergonomics, v. 21, n. 3 , p. 231-233.

Yoshitake, H. (1971). Relations between the symptoms and feeling of fatigue. Ergonomics, v. 14, n. 1, p. 175-186.

\section{Anexo: ESCALA DE AVALIAÇÃO DA FADIGA}

Intruções. Abaixo são apresentadas dez afirmações acerca de como você pode se sentir no seu dia-adia. Para cada afirmação você deve escolher uma das cinco alternativas de resposta que se apresentam ao lado, variando de $\mathbf{1}=$ Nunca a $\mathbf{5}=$ Sempre.

(5 = Sempre; 4 = Frequentemente; 3 = Algumas vezes; $\quad 2$ = Raramente $; 1=$ Nunca $)$

01._ Sinto-me incomodado devido à fadiga.

02.___ Fico cansado muito rapidamente.

03.___ Não faço muitas coisas durante o dia

04.__ Tenho suficiente energia para o meu dia-a-dia.

05.___ Sinto-me exausto fisicamente..

06.___ Tenho problemas para começar coisas..

07.__ Tenho problemas em pensar claramente..

08.___ Não sinto vontade de fazer nada.

09.___ Sinto-me exausto mentalmente.

10.__ Posso me concentrar bem quando estou fazendo algo.

Sobre os autores:

(1) Gislene Farias de Oliveira é Psicóloga e professora adjunta da Universidade Regional do Cariri URCA e Universidade Federal do Ceará - UFC.

E-mail: gislenefo@hotmail.com

(2) Valdiney Veloso Gouveia é Psicólogo e professor adjunto da Universidade Federal da Paraíba UFPB.

E-mail: vvgouveia@gmail.com 
(3) Ginna Pereira Peixoto é Psicóloga pela Universidade Federal da Paraíba, especialista em Saúde da Família pela Faculdades Integradas de Patos - PB.

E-mail: ginnapp@ig.com.br

(4) Maria Alinele Lucena Soares é Pedagoga pela Universidade Regional do Cariri - URCA e Licenciada em Língua Portuguesa pela Universidade Vale do Acaraú - UVA. Mestranda em Ciências da Educação pela Universidad San Carlos - Paraguai.

E-mail: alinelelucena@hotmail.com

\section{Como citar este artigo (Formato ISO):}

OLIVEIRA, G. F.; GOUVEIA, V. V.; PEIXOTO, G. P.; SOARES, M. A. L. Análise fatorial da escala de avaliação da fadiga em uma amostra de universitários de instituição pública. Id on Line Revista de Psicologia, Julho de 2010, vol.1, no.11, p.51-60. ISSN 1981-1189. 